\title{
STRATIFICATION OF CONTINUOUS MAPS OF AN INTERVAL
}

\author{
L. S. BLOCK AND W. A. COPPEL
}

\begin{abstract}
We define the notion of turbulence for a continuous map of an interval into the line and study its relation with periodic and homoclinic points. We define also strongly simple orbits and show, in particular, that they represent periodic orbits with minimum entropy. Further results are obtained for unimodal maps with negative Schwarzian, which sharpen recent results of Block and Hart.
\end{abstract}

1. Introduction. Throughout the paper $f: I \rightarrow \mathbf{R}$ will denote a continuous map of an interval into the real line. For definiteness we will suppose that the interval $I$ is compact, although this restriction will usually not be essential. If $J, K$ are compact subintervals of $I$ we write $J \rightarrow K$ if $K \subseteq f(J)$. It is well known-see, for example, Li et al. [15] - that if $J_{1}, \ldots, J_{m}$ are compact subintervals such that $J_{1} \rightarrow \cdots \rightarrow J_{m}$ $\rightarrow J_{1}$, then there exists a point $x$ such that $f^{m}(x)=x$ and $f^{k-1}(x) \in J_{k}$ for $1 \leqslant k \leqslant m$.

A point $x$ is said to be periodic with period $n$ if $f^{n}(x)=x$ and $f^{k}(x) \neq x$ for $0<k<n$. The orbit of $x$ is then the set of $n$ distinct points $x, f(x), \ldots, f^{n-1}(x)$. A fixed point of $f$ is a periodic point with period 1 .

For the given interval $I$, let $\mathscr{P}_{n}$ denote the set of all continuous maps $f: I \rightarrow \mathbf{R}$ such that $f$ has a periodic point of period $n$. A remarkable theorem of Šarkovskii [19] states that

$$
\begin{aligned}
\mathscr{P}_{3} & \subset \mathscr{P}_{5} \subset \mathscr{P}_{7} \subset \cdots \subset \mathscr{P}_{6} \subset \mathscr{P}_{10} \subset \cdots \subset \mathscr{P}_{12} \subset \mathscr{P}_{20} \\
& \subset \cdots \subset \mathscr{P}_{8} \subset \mathscr{P}_{4} \subset \mathscr{P}_{2} \subset \mathscr{P}_{1} .
\end{aligned}
$$

This ordering provides a stratification of the space $C(I, \mathbf{R})$ of all continuous maps from $I$ to $\mathbf{R}$.

In $\$ 2$ we define the concept of turbulence and use it to obtain a refinement of the Šarkovskii stratification. Although this concept has appeared implicitly in the literature (see $[2,8,14])$, we believe that its significance has not been sufficiently recognized.

In $\S 3$ we define the notion of strongly simple orbit and show that it arises naturally in connection with the turbulence stratification and in the study of topological

Received by the editors November 9, 1984 and, in revised form, November 4, 1985.

1980 Mathematics Subject Classification (1985 Revision). Primary 54H20, 26A18; Secondary 54C70. $58 \mathrm{~F} 20$.

Key words and phrases. Šarkovskii stratification, turbulence, strongly simple orbit, homoclinic point. unimodal map.

The first author was Visiting Fellow at the Australian National University during the preparation of this paper. 
entropy. We also extend a result of Block and Hart [7] by showing that if a map has an orbit of period $n$, then it has a strongly simple orbit of period $n$.

In $\$ 4$ we study the relationship between turbulence and the existence of homoclinic points. We introduce two special types of homoclinic point, one orientationpreserving and the other orientation-reversing. Although there exist homoclinic points which are of neither type, any map with a homoclinic point must have a homoclinic point of one of these two types. However, the real justification for concentrating attention on these two types lies in the simple and precise results which are obtained.

In $\$ 5$ we consider the important special case of unimodal maps with negative Schwarzian. Our results here sharpen those of Block and Hart [7] and place them in a more general setting.

We also make a notational innovation. We denote by $\langle a, b\rangle$ the closed interval with endpoints $a$ and $b$, when we do not know (or care) whether $a \lessgtr b$.

2. Turbulence. A continuous map $f: I \rightarrow \mathbf{R}$ will be said to be turbulent if there exist compact subintervals $J, K$ of $I$ with at most one common point such that

$$
J \cup K \subseteq f(J) \cap f(K) .
$$

This terminology was suggested by Theorem 1 of Lasota and Yorke [14]. It follows at once from the definition that if $f$ is turbulent, then $f^{n}$ is also turbulent for every $n>1$.

It is evident that the map $f$ is turbulent if there exist points $a, b, c \in I$ such that

$$
f(b)=f(a)=a, \quad f(c)=b,
$$

and either $a<c<b$ or $b<c<a$. Our first result shows that this sufficient condition for turbulence is also necessary.

LEMMA 1. If $f$ is turbulent there exist points $a, b, c \in I$ such that (2) holds and, in addition, either

$$
\begin{gathered}
a<c<b, \\
f(x)>a \text { for } a<x<b, \\
x<f(x)<b \text { for } a<x<c,
\end{gathered}
$$

or the same with all inequalities in (3) reversed.

Proof. Let $J=[\alpha, \beta]$ and $K=[\gamma, \delta]$, where $\beta \leqslant \gamma$, be compact subintervals such that (1) holds. If $\beta=\gamma$ we may assume that $f(\beta) \neq \beta$, since otherwise we can choose $J_{0} \subset J$ so that $f\left(J_{0}\right)=f(J)$ and $J_{0} \cap K=\varnothing$.

Let $a^{\prime}$ be the least fixed point of $f$ in $J$ and let $b^{\prime}$ be the greatest point of $K$ for which $f\left(b^{\prime}\right)=a^{\prime}$. Suppose first that $f\left(c^{\prime}\right)=b^{\prime}$ for some $c^{\prime} \in\left(a^{\prime}, b^{\prime}\right)$. Then we can take $a$ to be the greatest fixed point of $f$ in $\left[a^{\prime}, c^{\prime}\right), b$ to be the least point of $\left(c^{\prime}, b^{\prime}\right]$ for which $f(b)=a$, and $c$ to be the least point of $\left(a, c^{\prime}\right]$ for which $f(c)=b$.

Suppose next that $f(x)<b^{\prime}$ for $a^{\prime} \leqslant x \leqslant b^{\prime}$. Then $f$ takes the value $\delta$ in the intervals $\left[\alpha, a^{\prime}\right)$ and $\left(b^{\prime}, \delta\right]$, and $f(x)>x \geqslant \alpha$ for $\alpha \leqslant x<a^{\prime}$. Thus $f\left(a^{\prime \prime}\right)=a^{\prime \prime}$ for some $a^{\prime \prime} \in\left(b^{\prime}, \delta\right], f\left(b^{\prime \prime}\right)=a^{\prime \prime}$ for some $b^{\prime \prime} \in\left[\alpha, a^{\prime}\right)$, and $f\left(c^{\prime \prime}\right)=b^{\prime \prime}$ for some $c^{\prime \prime} \in\left(a^{\prime}, \beta\right]$. Then, as before, we can take $a$ to be the least fixed point of $f$ in 
( $\left.c^{\prime \prime}, a^{\prime \prime}\right], b$ to be the greatest point of $\left[b^{\prime \prime}, c^{\prime \prime}\right)$ for which $f(b)=a$, and $c$ to be the greatest point of $\left[c^{\prime \prime}, a\right)$ for which $f(c)=b$. Q.E.D.

We study next the relation between turbulence and the existence of periodic points.

LEMMA 2. If $f$ is turbulent, then $f$ has periodic points of all periods.

Proof. Let $J, K$ be compact subintervals with at most one common point such that (1) holds. By Lemma 1 we may assume that if $J$ and $K$ are not disjoint then their common point is not periodic. Since $J \rightarrow K \rightarrow \cdots \rightarrow K \rightarrow J$, for any given $n \geqslant 1$ there exists a point $x \in J$ such that $f^{n}(x)=x$ and $f^{k}(x) \in K$ for $0<k<n$. Evidently $x$ has period $n$. Q.E.D.

The following result is an immediate consequence of Li et al. [15] and Šarkovskii's theorem.

TheOREM A. If, for some odd $n>1$, there exists a point $x \in I$ such that either $f^{n}(x) \leqslant x<f(x)$ or $f(x)<x \leqslant f^{n}(x)$, then $f$ has a periodic point of period $n$.

This result will be used in the proof of Lemma 3 and also later.

LEMMA 3. Let $n>1$ be odd. Then $f$ has an orbit of period $n$ if and only if $f^{n}$ is turbulent, and in this case $f^{2}$ is also turbulent.

Proof. Suppose first that $f$ has an orbit of period $n$. By a result of Block and Hart, which is stated below as Theorem B in $\S 3$, we may assume that this orbit has the form

$$
f^{n-1}(x)<\cdots<f^{2}(x)<x<f(x)<\cdots<f^{n-2}(x) .
$$

If we put $J=[x, f(x)], K=\left[f^{2}(x), x\right]$ then

$$
J \cup K \subseteq f(J) \cap f^{n-1}(K)
$$

and hence $f^{n}$ is turbulent. On the other hand, if we put $J=\left[f^{n-1}(x), f^{n-3}(x)\right]$, $K=\left[f^{n-3}(x), f(x)\right]$ then

$$
J \cup K \subseteq f^{2}(J) \cap f^{2}(K)
$$

and thus $f^{2}$ is turbulent.

Suppose next that $f^{n}$ is turbulent. By Lemma 1 we may assume that there exist points $a<c<b$ such that $f^{n}(x)>a$ for $a<x<b$ and $f^{n}(b)=f^{n}(a)=a$, $f^{n}(c)=b$. If $f(a) \neq a$, then $f$ has a point of period $n$ by Šarkovskii's theorem. Thus we may assume that $f(a)=a$. If $f(c)<c$ or if $f(b)>b$, then $f$ has a point of period $n$ by Theorem A. Thus we may assume that $f(c)>c$ and $f(b)<b$. We must actually have $f(b) \leqslant a$, since $f(b) \in(a, b)$ would imply $a=f^{n+1}(b)>a$. It follows that we must also have $f(c) \geqslant b$, since $f(c) \in(c, b)$ would imply $f(b)=$ $f^{n+1}(c)>a$. Thus $[a, b] \subseteq f[a, c] \cap f[c, b]$ and we can now apply Lemma 2 . Q.E.D.

Any positive integer $n$ can be uniquely represented in the form $n=q m$, where $q \geqslant 1$ is odd and $m=2^{s}$ for some $s \geqslant 0$. Throughout the rest of the paper $q, m$, and $s$ will be defined in terms of $n$ in this way. 
LEMMA 4. Suppose $n$ is not a power of 2, i.e. $n=q m$ with $q>1$. Then $f$ has an orbit of period $n$ if and only if $f^{n}$ is turbulent, and in this case $f^{2 m}$ is also turbulent.

This follows at once from Lemma 3, using Šarkovskii's theorem and the relation between the periods of a periodic point under $f$ and $f^{m}$ (Lemma 2 of [9]).

Let $\mathscr{T}_{n}$ denote the set of all continuous maps $f: I \rightarrow \mathbf{R}$ such that $f^{n}$ is turbulent. Then Lemma 4 states that $\mathscr{T}_{n}=\mathscr{P}_{n}$ if $n$ is not a power of 2 and, in conjunction with Lemma 2, that the Sarkovskii stratification admits the following refinement:

$$
\begin{aligned}
\mathscr{T}_{1} & \subset \mathscr{P}_{3} \subset \mathscr{P}_{5} \subset \cdots \subset \mathscr{T}_{2} \subset \mathscr{P}_{6} \subset \mathscr{P}_{10} \subset \cdots \subset \mathscr{T}_{4} \subset \mathscr{P}_{12} \\
& \subset \cdots \subset \mathscr{P}_{4} \subset \mathscr{P}_{2} \subset \mathscr{P}_{1} .
\end{aligned}
$$

(It is shown below that the inclusions are indeed strict.)

A result equivalent to this has been given by Blokh [8], using the notion of an $L$-scheme due to Sarkovskii [19]. We believe that the notion of turbulence is more natural and simplifies the proofs. Moreover we have found that its use clarifies and sharpens other results in the literature. For example, in Proposition 3.6 of $\mathrm{Li}$ et al [16], the conclusion can be strengthened from "there exists a periodic point of period 6" to " $f 2$ is turbulent".

It has been shown by Block [4] that each term of the Šarkovskii stratification is contained in the interior of its successors. Our next result shows that this remains true for the turbulence stratification.

LeMmA 5. $\mathscr{T}_{2^{s}} \subset$ int $\mathscr{P}_{3 \cdot 2^{s}}$ for any $s \geqslant 0$.

Proof. It is sufficient to give the proof for $s=0$, since the general case follows by Lemma 2 of [9]. Let $f: I \rightarrow \mathbf{R}$ be a continuous map which is turbulent. We wish to show that there is a neighborhood $\mathscr{N}$ of $f$ in $C(I, \mathbf{R})$ such that any $g \in \mathscr{N}$ has a periodic point of period 3.

By Lemma 1 we may assume that there are points $a<c<b$ with $f(b)=f(a)=a$ and $f(c)=b$. Then

$$
f^{3}(c)<c<f(c) .
$$

There exists a neighborhood $\mathscr{N}$ of $f$ such that the displayed inequalities continue to hold when $f$ is replaced by any $g \in \mathscr{N}$. Then $g$ has a periodic point of period 3 , by Theorem A. Q.E.D.

It is easily seen that any $C^{0}$-neighborhood of a map $f$ contains turbulent maps. Hence $\mathscr{T}_{n}$ is certainly not a closed subset of $C(I, \mathbf{R})$. However, we now show that we obtain a closed set if we restrict attention to continuously differentiable maps.

LEMMA 6. If the interval $I$ is compact, then $\mathscr{T}_{n}^{1}:=\mathscr{T}_{n} \cap C^{1}(I, \mathbf{R})$ is a closed subset of $C^{1}(I, \mathbf{R})$ for any $n>0$.

Proof. If $f \in \mathscr{T}_{n}^{1}$ then, by Lemma 1 , there exist points $a, b, c \in I$ such that $f^{n}(b)=f^{n}(a)=a, f^{n}(c)=b$, and either $a<c<b$ or $b<c<a$. If we put $g=f^{n}$ then, by the mean-value theorem of the differential calculus, the interval $\langle a, b\rangle$ contains a point $y$ such that $g^{\prime}(y)=0$ and a point $z$ such that $g^{\prime}(z)>1$. Here the notation $\langle a, b\rangle$ is used as defined in the introduction. 
Now suppose $f_{k} \in \mathscr{T}_{n}^{1}$ and $f_{k} \rightarrow f$ in $C^{1}(I, \mathbf{R})$ as $k \rightarrow \infty$. Without loss of generality we may assume that there exists points $a_{k}<c_{k}<b_{k}$ such that $f_{k}^{n}\left(b_{k}\right)=$ $f_{k}^{n}\left(a_{k}\right)=a_{k}, f_{k}^{n}\left(c_{k}\right)=b_{k}$. Moreover, by restriction to a subsequence we may assume that $a_{k} \rightarrow a, b_{k} \rightarrow b, c_{k} \rightarrow c$. Then $a \leqslant c \leqslant b$ and $f^{n}(b)=f^{n}(a)=a$, $f^{n}(c)=b$. It follows that also $f \in \mathscr{T}_{n}^{1}$, unless $a=c=b$. But the latter case cannot arise, since by applying the first part of the proof to $g_{k}=f_{k}^{n}$, we would then obtain in the limit both $g^{\prime}(a)=0$ and $g^{\prime}(a) \geqslant 1$. Q.E.D.

3. Strongly simple orbits. The notion of a simple periodic orbit was first introduced by Block [3] for the case in which the period is a power of 2 . The definition is inductive. Any fixed point is a simple orbit, and an orbit of $f$ with period $2^{s}$, where $s>0$, is simple if the left and right halves of the orbit are simple orbits of $f^{2}$ with period $2^{s-1}$.

Block and Hart [7], and independently Ho [13], extended this definition to the case in which the period is any positive integer. If $q>1$ is odd then an orbit of period $q$ with midpoint $x$ is simple if its points have either the order

$$
f^{q-1}(x)<f^{q-3}(x)<\cdots<f^{2}(x)<x<f(x)<\cdots<f^{q-2}(x)
$$

or the reverse order. (Attention was first focused on this order by Štefan [20].) If $n=q m$, where $q>1$ is odd and $m=2^{s}>1$, then an orbit of period $n$ is simple if it consists of $m$ blocks of $q$ consecutive points, each block forming a simple orbit of $g=f^{m}$ with period $q$, and the blocks themselves being permuted by $f$ like a simple orbit with period $m$.

Block and Hart [7] proved the following general result:

THEOREM B. If $f$ has an orbit of period $n$, then it has a simple orbit of period $n$.

Block [3] has proved another result involving simple orbits which we will use later:

THEOREM C. If, for some $s>1, f$ has an orbit of period $2^{s}$ which is not simple, then $f$ also has an orbit of period $3 \cdot 2^{s-2}$.

In the present section we introduce the notion of a strongly simple periodic orbit and show its significance. Any simple orbit of odd period or of period a power of 2 is said to be strongly simple. A simple orbit of period $n=q m$, where $q>1$ and $m>1$, is said to be strongly simple if $f$ maps the midpoint of every block of $q$ consecutive points into another such midpoint, with one exception. This implies that $f$ maps every block monotonically onto another block, with one exception.

With this terminology a result due to Coppel [9] (cf. also Ho [12]) can be stated in the following way.

THEOREM D. If $f \in \mathscr{P}_{n}$, but $f \notin \mathscr{P}_{k}$ whenever $\mathscr{P}_{k} \subset \mathscr{P}_{n}$ and $1<k<n$, then every orbit of $f$ of period $n=q m$ is strongly simple unless $q=3$, in which case every orbit of $f$ of period $n$ is simple.

For $q=1$, Theorem $\mathrm{D}$ reduces to Theorem $\mathrm{C}$. Our results here will provide an explanation of why $q=3$ is exceptional. Moreover we will show that Theorem B continues to hold if in the conclusion "simple" is replaced by "strongly simple", and 
that the strongly simple orbits with period $n$ represent those with minimum topological entropy.

PROPOSITION 1. If $f$ has an orbit of period $3 m$ which is not strongly simple, where $m=2^{s}>1$, then $f^{m}$ is turbulent.

Proof. If some orbit of period $3 m$ is not simple, then $f$ has an orbit of period $q m / 2$ for some odd $q>1$, by Theorem $\mathrm{D}$, and hence $f^{m}$ is turbulent, by Lemma 4 . We may therefore assume that all orbits of period $3 m$ are simple.

Consider one such orbit. For some block $B=\left\{y_{1}, y_{2}, y_{3}\right\}$ with midpoint $y_{2}$ we must have $f\left(y_{2}\right) \neq z_{2}$, where $z_{2}$ is the midpoint of the block $f(B)=\left\{z_{1}, z_{2}, z_{3}\right\}$. We may suppose the notation chosen so that $f\left(y_{2}\right)=z_{1}, f\left(y_{1}\right)=z_{2}, f\left(y_{3}\right)=z_{3}$. Then $f\left\langle y_{2}, y_{3}\right\rangle \supseteq\left\langle z_{1}, z_{3}\right\rangle$ and hence $f^{m}\left\langle y_{2}, y_{3}\right\rangle \supseteq\left\langle y_{1}, y_{3}\right\rangle$. If $f^{k}\left(y_{1}\right)$ and $f^{k}\left(y_{2}\right)$ are both endpoints of $f^{k}(B)$ for some $k$ such that $1<k \leqslant m$, then also $f^{m}\left\langle y_{1}, y_{2}\right\rangle$ $\supseteq\left\langle y_{1}, y_{3}\right\rangle$ and hence $f^{m}$ is turbulent. We may therefore assume that for each $k$ with $0<k \leqslant m$ either $f^{k}\left(y_{1}\right)$ or $f^{k}\left(y_{2}\right)$ is the midpoint of $f^{k}(B)$, and hence $f^{k}\left(y_{3}\right)=f^{k-1}\left(z_{3}\right)$ is an endpoint of $f^{k}(B)$. In particular this implies that

$$
f^{m}\left(y_{3}\right)=f^{m-1}\left(z_{3}\right)=y_{1}, \quad f^{m}\left(y_{1}\right)=f^{m-1}\left(z_{2}\right)=y_{2}, \quad f^{m}\left(y_{2}\right)=f^{m-1}\left(z_{1}\right)=y_{3},
$$

and hence $f^{m}\left\langle z_{1}, z_{2}\right\rangle \supseteq f\left\langle y_{2}, y_{3}\right\rangle \supseteq\left\langle z_{1}, z_{3}\right\rangle$. If the orbit is not strongly simple there is a least $k$ with $1<k \leqslant m$ such that $f^{k-1}\left(z_{2}\right)$ is not the midpoint of $f_{k}(B)$. Then $f^{k-1}\left\langle z_{2}, z_{3}\right\rangle \supseteq f_{k}(B)$ and hence $f^{m}\left\langle z_{2}, z_{3}\right\rangle \supseteq\left\langle z_{1}, z_{3}\right\rangle$. Thus again $f^{m}$ is turbulent. Q.E.D.

It is easily shown by examples that Proposition 1 no longer holds if in its statement we replace $3 m$ by $q m$, with an arbitrary odd $q>3$. The converse of Proposition 1 is also false, since a map may have an orbit of odd period $q>1$, although every orbit of period 6 is strongly simple. A simple example is the piecewise linear map defined by $f(0)=1, f(1)=2, f(2)=0$.

Proposition 2. If $f \in \mathscr{T}_{n}$, but $f \notin \mathscr{T}_{k}$ whenever $\mathscr{T}_{k} \subset \mathscr{T}_{n}$ and $1<k<n$, then every orbit of $f$ of period $n$ is strongly simple.

Proof. If $q>1$ then $\mathscr{T}_{n}=\mathscr{P}_{n}$, by Lemma 4 . Thus the result follows from Proposition 1 if $q=3$ and from Theorem $\mathrm{D}$ if $q>3$. If $q=1$ then the result is trivial for $s=0,1$ and it follows from Theorem $\mathrm{C}$ if $s>1$. Q.E.D.

Thus the exceptional case in Theorem D arises from considering the Sarkovskii stratification, instead of the turbulence stratification.

Proposition 3. If $f$ has an orbit of period $3 m$, where $m=2^{s}>1$, then $f$ has a strongly simple orbit of period $3 \mathrm{~m}$.

Proof. By Theorem B, $f$ has a simple orbit of period $3 m$. Let the blocks $B_{1}, \ldots, B_{m}$ of this orbit be numbered so that $f\left(B_{k}\right)=B_{k+1}(1 \leqslant k<m), f\left(B_{m}\right)=$ $B_{1}$. If $b_{k}$ denotes the midpoint of $B_{k}$, we may choose the numbering so that in addition $f\left(b_{m}\right) \neq b_{1}$. Furthermore, if $B_{k}=\left\{a_{k}, b_{k}, c_{k}\right\}$ we may choose the notation so that $f\left(a_{m}\right)=b_{1}, f\left(b_{m}\right)=c_{1}, f\left(c_{m}\right)=a_{1}$. 
If the orbit is not strongly simple there is a greatest positive integer $h<m$ such that $f\left(b_{h}\right) \neq b_{h+1}$. Let $a_{h}$ denote the endpoint of $B_{h}$ such that $f\left(a_{h}\right)=b_{h+1}$. Also, if $h<m-1$ let $a_{k}, c_{k}$ denote the endpoints of $B_{k}$ such that $f^{m-k}\left(a_{k}\right)=a_{m}$, $f^{m-k}\left(c_{k}\right)=c_{m}(h<k<m)$. There are two cases to consider, according as $f\left(b_{h}\right)=$ $c_{h+1}$ or $a_{h+1}$. We restrict attention to the first case, the argument in the second case being similar. Thus we have $f\left(a_{h}\right)=b_{h+1}, f\left(b_{h}\right)=c_{h+1}, f\left(c_{h}\right)=a_{h+1}$. If $f^{h-1}\left(c_{1}\right)$ $=a_{h}$ then $f^{h}\left(c_{1}\right)=b_{h+1}, f^{m-1}\left(c_{1}\right)=b_{m}$, and $f^{m}\left(c_{1}\right)=c_{1}$, which is impossible. It follows that there are just two possibilities:

(i) $f^{h-1}\left(c_{1}\right)=c_{h}, f^{h-1}\left(a_{1}\right)=a_{h}, f^{h-1}\left(b_{1}\right)=b_{h}$;

(ii) $f^{h-1}\left(c_{1}\right)=b_{h}, f^{h-1}\left(a_{1}\right)=c_{h}, f^{h-1}\left(b_{1}\right)=a_{h}$.

Suppose first that (i) holds. Choose $d_{h} \in\left\langle b_{h}, c_{h}\right\rangle$ so that $f\left(d_{h}\right)=b_{h+1}$. Then choose $d_{1} \in\left\langle b_{1}, c_{1}\right\rangle$ so that $d_{k}:=f^{k-1}\left(d_{1}\right) \in\left\langle f^{k-1}\left(b_{1}\right), f^{k-1}\left(c_{1}\right)\right\rangle$ for $1<k<h$ and $d_{h}=f^{h-1}\left(d_{1}\right)$. Choose $d_{m} \in\left\langle a_{m}, b_{m}\right\rangle$ so that $f\left(d_{m}\right)=d_{1}$. Choose $d_{h+1} \in$ $\left\langle a_{h+1}, b_{h+1}\right\rangle$ so that $d_{k}:=f^{k-h-1}\left(d_{h+1}\right) \in\left\langle a_{k}, b_{k}\right\rangle$ for $h+1<k<m$ and $d_{m}=$ $f^{m-h-1}\left(d_{h+1}\right)$. Choose $e_{h} \in\left\langle d_{h}, c_{h}\right\rangle$ so that $f\left(e_{h}\right)=d_{h+1}$. Finally choose $e_{1} \in$ $\left\langle d_{1}, c_{1}\right\rangle$ so that $e_{k}:=f^{k-1}\left(e_{1}\right) \in\left\langle d_{k}, f^{k-1}\left(c_{1}\right)\right\rangle$ for $1<k<h$ and $e_{h}=f^{h-1}\left(e_{1}\right)$. For $1 \leqslant k \leqslant h$ put

$$
R_{k}=\left\langle f^{k-1}\left(b_{1}\right), d_{k}\right\rangle, \quad S_{k}=\left\langle d_{k}, e_{k}\right\rangle, \quad T_{k}=\left\langle e_{k}, f^{k-1}\left(c_{1}\right)\right\rangle,
$$

and for $h<k \leqslant m$ put

$$
R_{k}=\left\langle b_{k}, c_{k}\right\rangle, \quad S_{k}=\left\langle d_{k}, b_{k}\right\rangle, \quad T_{k}=\left\langle a_{k}, d_{k}\right\rangle .
$$

Then

$$
R_{1} \rightarrow \cdots \rightarrow R_{m} \rightarrow S_{1} \rightarrow \cdots \rightarrow S_{m} \rightarrow T_{1} \rightarrow \cdots \rightarrow T_{m} \rightarrow R_{1},
$$

and hence $f$ has a strongly simple orbit of period $3 m$.

Suppose next that (ii) holds. Choose $d_{1} \in\left\langle a_{1}, b_{1}\right\rangle$ so that $d_{k}:=f^{k-1}\left(d_{1}\right) \in$ $\left\langle f^{k-1}\left(a_{1}\right), f^{k-1}\left(b_{1}\right)\right\rangle$ for $1<k<h$ and $b_{h}=f^{h-1}\left(d_{1}\right)$. Then choose $d_{m} \in\left\langle b_{m}, c_{m}\right\rangle$ so that $f\left(d_{m}\right)=d_{1}$. Choose $d_{h} \in\left\langle b_{h}, c_{h}\right\rangle$ so that $d_{k}:=f^{k-h}\left(d_{h}\right) \in\left\langle b_{k}, c_{k}\right\rangle$ for $h<k<m$ and $d_{m}=f^{m-h}\left(d_{h}\right)$. Choose $e_{1} \in\left\langle a_{1}, d_{1}\right\rangle$ so that $e_{k}:=f^{k-1}\left(e_{1}\right) \in$ $\left\langle f^{k-1}\left(a_{1}\right), d_{k}\right\rangle$ for $1<k<h$ and $d_{h}=f^{h-1}\left(e_{1}\right)$. Choose $e_{m} \in\left\langle d_{m}, c_{m}\right\rangle$ so that $f\left(e_{m}\right)=e_{1}$. Finally choose $e_{h+1} \in\left\langle d_{h+1}, c_{h+1}\right\rangle$ so that $e_{k}:=f^{k-h-1}\left(e_{h+1}\right) \in$ $\left\langle d_{k}, c_{k}\right\rangle$ for $h+1<k<m$ and $e_{m}=f^{m-h-1}\left(e_{h+1}\right)$. Put

$$
\begin{gathered}
R_{k-h}=\left\langle b_{k}, d_{k}\right\rangle, \quad S_{k-h}=\left\langle d_{k}, e_{k}\right\rangle, \quad T_{k-h}=\left\langle e_{k}, c_{k}\right\rangle \quad(h<k \leqslant m), \\
R_{m-h+k}=\left\langle d_{k}, f^{k-1}\left(b_{1}\right)\right\rangle, \quad S_{m-h+k}=\left\langle e_{k}, d_{k}\right\rangle, \quad T_{m-h+k}=\left\langle f^{k-1}\left(a_{1}\right), e_{k}\right\rangle \\
\quad(1 \leqslant k<h), \\
R_{m}=\left\langle a_{h}, b_{h}\right\rangle, \quad S_{m}=\left\langle b_{h}, d_{h}\right\rangle, \quad T_{m}=\left\langle d_{h}, c_{h}\right\rangle .
\end{gathered}
$$

Then

$$
R_{1} \rightarrow \cdots \rightarrow R_{m} \rightarrow S_{1} \rightarrow \cdots \rightarrow S_{m} \rightarrow T_{1} \rightarrow \cdots \rightarrow T_{m} \rightarrow R_{1},
$$

and it follows again that $f$ has a strongly simple orbit of period $3 m$. Q.E.D.

PROPOSITION 4. If $f$ has a strongly simple orbit of period $n=q m$, where $q>1$, then $f$ also has a strongly simple orbit of period $(q+2) m$. 
Proof. The strongly simple orbit of period $n$ consists of $m$ blocks $B(1), \ldots, B(m)$ of $q$ consecutive points. If we put $g=f^{m}$, the points of each block have either the order

$$
z_{q}<z_{q-2}<\cdots<z_{3}<z_{1}<z_{2}<\cdots<z_{q-3}<z_{q-1}
$$

or the reverse order, where $z_{1}$ is the midpoint of the block and $z_{j}=g^{j-1}\left(z_{1}\right)$ $(1<j \leqslant q)$. More precisely, we will write $z_{j}(k)$ in place of $z_{j}$ to denote that the point belongs to the block $B(k)$. Furthermore the blocks can be numbered so that

$$
f\left(z_{1}(k)\right)=z_{1}(k+1) \text { for } 1 \leqslant k<m \text {. }
$$

Then

$$
\begin{gathered}
f\left(z_{j}(k)\right)=z_{j}(k+1) \text { for } 1 \leqslant k<m, 1 \leqslant j \leqslant q, \\
f\left(z_{j}(m)\right)=z_{j+1}(1) \text { for } 1 \leqslant j<q, \\
f\left(z_{q}(m)\right)=z_{1}(1) .
\end{gathered}
$$

Since $f\left\langle z_{1}(k), z_{2}(k)\right\rangle \supseteq\left\langle z_{1}(k+1), z_{2}(k+1)\right\rangle$ for $1 \leqslant k<m$ and $f\left\langle z_{1}(m), z_{2}(m)\right\rangle \supseteq\left\langle z_{3}(1), z_{2}(1)\right\rangle$, there exists a point $w$ such that $f^{m}(w)=w$ and $f^{k-1}(w) \in\left\langle z_{1}(k), z_{2}(k)\right\rangle$ for $1 \leqslant k \leqslant m$. Similarly there exists a point $x$ such that $f^{m}(x)=z_{1}(1)$ and $f^{k-1}(x) \in\left\langle f^{k-1}(w), z_{2}(k)\right\rangle$ for $1 \leqslant k \leqslant m$. Finally there exists a point $y$ such that $f^{m}(y)=x$ and $f^{k-1}(y) \in\left\langle z_{1}(k), f^{k-1}(w)\right\rangle$ for $1 \leqslant k \leqslant m$. For $1 \leqslant k \leqslant m$ put

$$
\begin{gathered}
J_{1}(k)=\left\langle f^{k-1}(y), f^{k-1}(w)\right\rangle, \quad J_{2}(k)=\left\langle f^{k-1}(w), f^{k-1}(x)\right\rangle, \\
J_{3}(k)=\left\langle z_{1}(k), f^{k-1}(y)\right\rangle, \quad J_{4}(k)=\left\langle f^{k-1}(x), z_{2}(k)\right\rangle, \\
J_{i}(k)=\left\langle z_{i-4}(k), z_{i-2}(k)\right\rangle, \quad(5 \leqslant i \leqslant q+2) .
\end{gathered}
$$

Then

$$
\begin{aligned}
J_{1}(1) \rightarrow & J_{1}(2) \rightarrow \cdots \rightarrow J_{1}(m) \\
\rightarrow & J_{2}(1) \rightarrow J_{2}(2) \rightarrow \cdots \rightarrow J_{2}(m) \\
& \quad \cdots \\
& \rightarrow J_{q+2}(1) \rightarrow J_{q+2}(2) \rightarrow \cdots \rightarrow J_{q+2}(m) \rightarrow J_{1}(1),
\end{aligned}
$$

and hence $f$ has a strongly simple orbit of period $(q+2) m$. Q.E.D.

We can now deduce without difficulty the main result of this section.

THEOREM 1. If $f$ has an orbit of period $n$, then it has a strongly simple orbit of period $n$.

Proof. By Theorem B, $f$ has a simple orbit of period $n$. If $n$ is odd or a power of 2 , then by definition this orbit is also strongly simple. Thus we may suppose $q>1$ and $m>1$ in the representation $n=q m$. If $f$ has an orbit of period $3 m$ then it has a strongly simple orbit of period $3 m$, by Proposition 3, and hence a strongly simple orbit of period $q m$, by Proposition 4. If $f$ does not have an orbit of period $3 m$ then, for some odd $r$ such that $3<r \leqslant q, f$ has an orbit of period $r m$ but no orbit of period $(r-2) m$. The orbit of period $r m$ is strongly simple, by Theorem $\mathrm{D}$, and hence $f$ has a strongly simple orbit of period $q m$, by Proposition 4 again. Q.E.D. 
We are also led to strongly simple orbits by the study of topological entropy. This notion was introduced by Adler et al. [1] and is further discussed by Denker et al. [10]. The topological entropy of a continuous map $f$ is denoted by $h(f)$. For any positive integer $m$ we have $h\left(f^{m}\right)=m h(f)$. It follows from Block et al. [5, Lemma 1.5] that if $f$ is turbulent, then $h(f) \geqslant \log 2$. Hence if $f^{m}$ is turbulent, then $h(f) \geqslant(\log 2) / m$.

Let $x_{1}<\cdots<x_{n}$ be an orbit of $f$ of period $n$. Corresponding to this orbit there is a directed graph with its associated adjacency matrix and characteristic polynomial. It follows also from Block et al. [5, Lemma 1.5] that $h(f) \geqslant \log \rho$, where $\rho$ is the largest positive root of the characteristic polynomial. Moreover, by Misiurewicz and Szlenk [17, Theorem 1], this lower bound is attained if $I=\left[x_{1}, x_{n}\right]$ and $f$ is strictly monotonic on each subinterval $\left[x_{j}, x_{j+1}\right](1 \leqslant j<n)$.

Block et al. [5] have shown in this way that if $f$ has an orbit of period $n=q m$, where $q>1$ is odd and $m=2^{s}$, then $h(f) \geqslant\left(\log \lambda_{q}\right) / m$, where $\lambda_{q}$ is the unique positive root of the polynomial $L_{q}(\lambda)=\lambda^{q}-2 \lambda^{q-2}-1$. (Note that $L_{q}(\lambda)$ has at most one positive root, by Descartes' rule of signs, and $L_{q}(1)<0<L_{q}(2)$.) We wish to determine when this lower bound for $h(f)$ is attained.

Proposition 5. If $f$ has an orbit of period $n=q m$, where $q>1$, and if

$$
h(f)=\left(\log \lambda_{q}\right) / m,
$$

then the orbit of period $n$ is strongly simple.

Conversely, suppose $f$ has a strongly simple orbit $x_{1}<\cdots<x_{n}$ of period $n=q m$, where $q>1$. Then (4) holds if $I=\left[x_{1}, x_{n}\right]$ and $f$ is strictly monotonic on each subinterval $I_{j}=\left[x_{j}, x_{j+1}\right](1 \leqslant j<n)$.

PRoof. For any odd $r<q$ we have $\lambda_{r}>\lambda_{q}$, since

$$
L_{q}\left(\lambda_{r}\right)=\lambda_{r}^{q-r}\left(\lambda_{r}^{r}-2 \lambda_{r}^{r-2}\right)-1=\lambda_{r}^{q-r}-1>0 .
$$

It follows from (4) and the lower bound of Block et al. that $f$ cannot have an orbit of period $r m$. Also, for any odd $t>1$,

$$
\lambda_{t}^{2}=2+1 / \lambda_{t}^{t-2}>2>\lambda_{q} .
$$

It follows similarly that if $m>1$ then $f$ cannot have an orbit of period $t m / 2$. Thus $f$ satisfies the hypotheses of Theorem D. Hence the given orbit of period $n=q m$ is strongly simple if $q>3$. If $q=3$ and the orbit is not strongly simple then $f^{m}$ is turbulent, by Proposition 1 , and hence $h(f) \geqslant(\log 2) / m$, which contradicts (4). This proves the first statement of the proposition.

The second statement follows directly from the remarks made above. For, by Coppel [9, Theorem 1], in the case of a strongly simple orbit the largest positive root $\rho$ of the associated characteristic polynomial is precisely $\rho=\lambda_{q}^{1 / m}$. Q.E.D.

4. Homoclinic points. According to Block [2], a periodic point $z$ of period $n$ is homoclinic if there exists a point $y \neq z$ in the unstable manifold $W^{u}\left(z, f^{n}\right)$ such that $f^{k n}(y)=z$ for some $k>0$. 
Homoclinic points are, like turbulence, an indication of the complexity of a map. In this section we study the relationship between these two concepts. Let $H(n)$ denote the set of all continuous maps $f: I \rightarrow \mathbf{R}$ with a homoclinic point of period $n$. We first prove

LEMMA 7. $H(n) \subseteq \mathscr{T}_{2 n}$ for any $n>0$.

Proof. Let $f \in H(n)$. We may restrict attention to the case $n=1$, simply by replacing $f$ by $f^{n}$. Let $z$ be a homoclinic fixed point of $f$. If there exist points $x, y$ with $z<x<y$ or $y<x<z$ such that $f(x)=y, f(y)=z$, then $f$ is turbulent and hence $f^{2}$ is also turbulent. If there exist points $x_{1}, x_{2}, x_{3}, x_{4}$ with $x_{1}<x_{3}<z<x_{4}$ $<x_{2}$ or $x_{2}<x_{4}<z<x_{3}<x_{1}$ such that $f\left(x_{1}\right)=z$ and $f\left(x_{k}\right)=x_{k-1}(k=2,3,4)$, then $f^{2}$ is again turbulent, since $\left\langle z, x_{2}\right\rangle \subseteq f^{2}\left\langle z, x_{4}\right\rangle \cap f^{2}\left\langle x_{4}, x_{2}\right\rangle$. But, by Block and Hart [6, Lemma 1], at least one of these possibilities must occur. Q.E.D.

On the basis of Lemma 1 and the proof of Lemma 7 we now introduce two special types of homoclinic point. First, let $\mathscr{H}_{n}$ denote the set of all continuous maps $f$ : $I \rightarrow \mathbf{R}$ for which there exist points $a, b, c$ such that

$$
f^{n}(b)=f^{n}(a)=a, \quad f^{n}(c)=b,
$$

$a$ has period $n$, and either

$$
\begin{gathered}
a<c<b, \\
f^{n}(x)>a \text { for } a<x<b, \\
x<f^{n}(x)<b \text { for } a<x<c,
\end{gathered}
$$

or the same with all inequalities in (6) reversed. Then $a$ is a homoclinic point of period $n$, since $c \in W^{u}\left(a, f^{n}\right)$, and

$$
\mathscr{H}_{n} \subseteq \mathscr{T}_{n},
$$

since $\langle a, b\rangle \subseteq f^{n}\langle a, c\rangle \cap f^{n}\langle c, b\rangle$. We will say in this case that $a$ is a homoclinic point of type (I). By Lemma 1 we actually have

$$
\mathscr{H}_{1}=\mathscr{T}_{1} .
$$

Secondly, let $\mathscr{H}_{n}^{\#}$ denote the set of all continuous maps $f: I \rightarrow \mathbf{R}$ for which there exist points $x_{0}, \ldots, x_{4}$ such that

$$
f^{n}\left(x_{0}\right)=x_{0}, \quad f^{n}\left(x_{k}\right)=x_{k-1} \quad(1 \leqslant k \leqslant 4),
$$

$x_{0}$ has period $n$, and either

$$
\begin{gathered}
x_{1}<x_{3}<x_{0}<x_{4}<x_{2}, \\
f^{n}(x)<x_{0}<f^{2 n}(x) \text { for } x_{0}<x<x_{2}, \\
x<f^{2 n}(x)<x_{2} \quad \text { for } x_{0}<x<x_{4},
\end{gathered}
$$

or the same with all inequalities in (10) reversed. Then $x_{0}$ is a homoclinic point of period $n$ and

$$
\mathscr{H}_{n}^{\#} \subseteq \mathscr{T}_{2 n},
$$


since $\left\langle x_{0}, x_{2}\right\rangle \subseteq f^{2 n}\left\langle x_{0}, x_{4}\right\rangle \cap f^{2 n}\left\langle x_{4}, x_{2}\right\rangle$. We will say in this case that $x_{0}$ is a homoclinic point of type (II). It is readily seen that relations (9) and (10) imply

$$
\begin{aligned}
& x_{1}<f^{n}(x) \text { for } x_{0}<x<x_{2} \\
& x_{3}<f^{n}(x) \text { for } x_{0}<x<x_{4} \\
& x_{0}<f^{n}(x) \text { for } x_{1}<x<x_{0} \\
& x_{2}>f^{n}(x) \text { for } x_{3}<x<x_{0}
\end{aligned}
$$

regardless of whether $x_{0}$ has period exactly $n$.

We first study the sets $\mathscr{H}_{n}, \mathscr{H}_{n}^{\#}$ in the case where $n$ is a power of 2.

Proposition 6. $\mathscr{H}_{2^{s}} \subseteq \mathscr{H}_{2^{s+2}}$ for any $s \geqslant 0$.

Proof. Let $f \in \mathscr{H}_{1}$ and, without loss of generality, let $a, b, c$ be points satisfying the relations (5) and (6) with $n=1$.

Since $f[a, c] \supseteq[a, b]$, there is a greatest point $d \in(a, c)$ such that $f(d)=c$. Since $f^{2}[d, c] \supseteq[a, b] \supseteq[d, c], f^{4}$ has a fixed point in $[d, c]$. If $\alpha$ is the least, then $d<\alpha<c$. Assume $f^{2}(\alpha)=\alpha$. Then $f^{2}(d, \alpha) \supseteq(\alpha, b)$ and hence $f^{2}(e)=c$ for some $e \in(d, \alpha)$. Then $f^{3}[d, e] \supseteq[a, b] \supseteq[a, c]$ and hence $f^{4}[d, e] \supseteq[a, b] \supseteq[d, e]$. Thus $f^{4}$ has a fixed point in $[d, e]$, which contradicts the definition of $\alpha$. We conclude that $f^{2}(\alpha) \neq \alpha$, and so $\alpha$ has period 4 .

Since $f^{4}(d)=a<d$, we have $f^{4}(x)<x$ for $d \leqslant x<\alpha$. On the other hand, since $f^{2}(a, d) \supseteq(a, b) \supseteq(a, d)$, we have $f^{4}(a, d) \supseteq(a, b)$. Thus $\alpha=f^{4}(\beta)$ for some greatest $\beta \in(a, d)$. Similarly $\beta=f^{4}(\gamma)$ for some greatest $\gamma \in(d, \alpha)$. It now follows readily that $f \in \mathscr{H}_{4}$.

Thus the proposition holds for $s=0$. Assume that $s>0$ and it holds for all smaller values of $s$. If $f \in \mathscr{H}_{2^{s}}$, then $g=f^{2} \in \mathscr{H}_{2^{s-1}}$. Hence $g \in \mathscr{H}_{2^{s+1}}$, by the induction hypothesis, and it follows that $f \in \mathscr{H}_{2^{s+2}}$, by [9, Lemma 2]. Q.E.D.

To establish the analogous property of $\mathscr{H}_{n}^{\#}$ we make use of the following preliminary result.

LEMMA 8. Suppose there exist points $a<c<b$ such that

$$
\begin{gathered}
f(a)=a=f^{2}(b), \quad f^{2}(c)=b, \\
f^{2}(x)>a \text { for } a<x<b \\
x<f^{2}(x)<b \text { for } a<x<c .
\end{gathered}
$$

Then $f \in \mathscr{H}_{1}^{\#}$ if $f(\xi)<a$ for some $\xi \in(a, b)$ and $f \in \mathscr{H}_{1}$ otherwise.

Proof. The conditions (13) evidently imply that $f(x) \neq a$ for $a<x<b$. Suppose first that $f(x)<a$ for every $x \in(a, b)$. In particular, $f(c)<a$ and $f(b) \leqslant a$. If $x \in(f(c), a)$ then $x=f(y)$ for some $\cdot y \in(a, c)$ and hence $f(x)=f^{2}(y)$ satisfies $a<f(x)<b$. Consequently $f^{2}(x)<a$ if $x \in(f(c), a)$. Since $f^{3}(b)=a$, it follows that either $f(b)<f(c)$ or $f(b)=a$.

Consider first the case $f(b)<f(c)$. If we put $x_{0}=a, x_{1}=f(b), x_{2}=b, x_{3}=$ $f(c), x_{4}=c$, then relations (9) and (10) are satisfied with $n=1$. Thus $f \in \mathscr{H}_{1}^{\#}$. 
Consider next the case $f(b)=a$. For some greatest $d \in(f(c), a)$ we must have $f(d)=c$. If $x \in(d, a)$ then $z=f(x) \in(a, c)$. Since $f^{2}(x)=x$ would imply $f^{2}(z)$ $=z$ and $f^{2}(x)=f(c)$ would imply $f^{2}(z)=b$, it follows that $f(c)<f^{2}(x)<x$ for $d<x<a$. If we now put $x_{0}=a, x_{1}=b, x_{2}=f(c), x_{3}=c, x_{4}=d$, the relations (9) and (10), with the inequalities reversed, are satisfied with $n=1$. Thus again $f \in \mathscr{H}_{1}^{\#}$.

Suppose next that $f(x)>a$ for every $x \in(a, b)$. Since $f^{3}(b)=a$ the conditions (13) imply that $f(b) \notin(a, b]$. If $f(b)>b$ then $[a, f(b)] \subseteq f[a, b] \cap f[b, f(b)]$ and hence $f \in \mathscr{T}_{1}=\mathscr{H}_{1}$. The only remaining possibility is $f(b)=a$. Since $f(c)>a$ we must have either $f(c)>b$ or $a<f(c)<b$. But if $f(c)>b$ then

$$
[a, b] \subseteq f[a, c] \cap f[c, b]
$$

and if $a<f(c)<b$ then

$$
[a, b] \subseteq f[a, f(c)] \cap f[f(c), b] .
$$

Thus $f \in \mathscr{H}_{1}$ in every case. Q.E.D.

We can now derive the main result of this section. It establishes a precise relation between the set $\mathscr{T}_{n}$ and the sets $\mathscr{H}_{n}$ and $\mathscr{H}_{n}^{\#}$, when $n$ is a power of 2.

THEOREM 2. $\mathscr{T}_{2^{s}}=\mathscr{H}_{2^{s-1}}^{\#} \cup \mathscr{H}_{2^{s}}$ for any $s>0$.

Proof. The right side is certainly contained in the left by (7) and (11). Thus we need only show that the left side is contained in the right.

We show first that $\mathscr{T}_{1} \subseteq \mathscr{H}_{1}^{\#} \cup \mathscr{H}_{2}$. Let $f \in \mathscr{T}_{1}$. By Lemma 1 we may assume that there exist points $a, b, c$ which satisfy the relations (2) and (3). Since $f(c)>c$ and $f(b)<b$ we have $f(x)=x$ for some $x \in(c, b)$. Let $\xi$ be the least fixed point of $f^{2}$ in $(c, b)$. Then $f^{2}(x)<x<\xi$ for $c \leqslant x<\xi$. For some $d \in(a, c)$ we have $f(d)=c$ and thus $f^{2}(d)=b$. Hence $f^{2}(\eta)=\xi$ for some greatest $\eta \in(a, c)$. Then $f^{2}(x)<\xi$ for $\eta<x \leqslant c$. Similarly $f^{2}(\zeta)=\eta$ for some greatest $\zeta \in(c, \xi)$. Then $f^{2}(x)>\eta$ for $\zeta<x<\xi$. If $f(\xi) \neq \xi$ it follows at once that $f \in \mathscr{H}_{2}$. If $f(\xi)=\xi$ then, since $f(c)>\xi$, it follows from Lemma 8 (with the inequalities reversed) that $f \in \mathscr{H}_{1}^{\#}$. This establishes our claim.

Suppose next that $f \in \mathscr{T}_{2}$. By Lemma 1 we may assume that there exist points $a$, $b, c$ which satisfy relations (5) and (6) with $n=2$. If $f(a) \neq a$ then $a$ has period 2 and hence $f \in \mathscr{H}_{2}$. If $f(a)=a$ then $f \in \mathscr{H}_{1} \cup \mathscr{H}_{1}^{\#}$, by Lemma 8 , and hence $f \in \mathscr{H}_{1}^{\#} \cup \mathscr{H}_{2}$, by the previous part of the proof. This proves the theorem for $s=1$.

Suppose finally that $f \in \mathscr{T}_{2^{s+1}}$ for some $s>0$. Then $g=f^{2^{s}} \in \mathscr{T}_{2}$ and hence $g \in \mathscr{H}_{1}^{\#} \cup \mathscr{H}_{2}$. If $g \in \mathscr{H}_{2}$ then $f \in \mathscr{H}_{2^{s+1}}$. If $g \in \mathscr{H}_{1}^{\#}$ we may suppose that relations (9) and (10) hold with $n=2^{s}$. If $f^{n / 2}\left(x_{0}\right) \neq x_{0}$ then $f \in \mathscr{H}_{2^{s}}^{\#}$. Thus to complete the proof we will assume $f^{n / 2}\left(x_{0}\right)=x_{0}$ and deduce a contradiction. By (10) we now have $f^{n / 2}(x) \neq x_{0}$ for $x_{0}<x<x_{2}$. If $f^{n / 2}(x)>x_{0}$ for $x_{0}<x<x_{2}$ then $f^{n}(x)>x_{0}$ for $x>x_{0}$ and $x$ close to $x_{0}$, which contradicts (10). Hence $f^{n / 2}(x)<x_{0}$ for $x_{0}<x<x_{2}$. Similarly from (12) we obtain $f^{n / 2}(x)>x_{0}$ for $x_{1}<x<x_{0}$. But then we again obtain $f^{n}(x)>x_{0}$ for $x>x_{0}$ and $x$ close to $x_{0}$. Q.E.D.

As a corollary we obtain the following counterpart to Proposition 6 . 
PROPOSITION 7. $\mathscr{H}_{1} \subseteq \mathscr{H}_{1}^{\#} \cup \mathscr{H}_{2}$ and $\mathscr{H}_{2^{s-1}}^{\#} \cup \mathscr{H}_{2^{s}} \subseteq \mathscr{H}_{2^{s}}^{\#} \cup \mathscr{H}_{2^{s+1}}$ for any $s>0$.

It is readily seen that Theorems 1-3 of Block and Hart [6] are contained in the preceding results, and some of our arguments have been based on theirs. The following examples show that our results are sharp:

If $f:[0,4] \rightarrow[0,4]$ is the piecewise linear map defined by

(i) $f(0)=0, f(2)=4, f\left(\frac{9}{4}\right)=\frac{19}{8}, f\left(\frac{15}{4}\right)=\frac{7}{4}, f(4)=0$, then $f \in \mathscr{H}_{1} \cap \mathscr{H}_{2}$ but $f \notin \mathscr{H}_{1}^{\#} \cup \mathscr{H}_{2}^{\#}$;

(ii) $f(0)=4, f(2)=2, f(3)=0, f(4)=2$, then $f \in \mathscr{H}_{1}^{\#} \cap \mathscr{H}_{2}^{\#}$ but $f \notin \mathscr{H}_{1} \cup$ $\mathscr{H}_{2} \cup \mathscr{H}_{4}$

(iii) $f(0)=4, f(2)=2, f(3)=0, f\left(\frac{15}{4}\right)=\frac{3}{4}, f(4)=2$, then $f \in \mathscr{H}_{1}^{\#} \cap \mathscr{H}_{4}$ but $f \notin \mathscr{H}_{1} \cup \mathscr{H}_{2} \cup \mathscr{H}_{2}^{\#}$

(iv) $f(0)=0, f(2)=4, f\left(\frac{5}{2}\right)=\frac{7}{4}, f\left(\frac{7}{2}\right)=\frac{3}{2}, f(4)=0$, then $f \in \mathscr{H}_{1} \cap \mathscr{H}_{1}^{\#}$ but $f \notin H(2)$;

(v) $f(0)=\frac{5}{2}, f(1)=4, f(3)=0, f(4)=\frac{1}{2}$, then $f \in \mathscr{H}_{2}^{\#}$ but $f \notin \mathscr{H}_{1} \cup \mathscr{H}_{1}^{\#} \cup$ $\mathscr{H}_{2} \cup \mathscr{H}_{4}$.

The relation between the sets $\mathscr{T}_{n}$ and $\mathscr{H}_{n}$, when $n$ is not a power of 2 , is even simpler.

THEOREM 3. $\mathscr{T}_{n}=\mathscr{H}_{n}$ if $n$ is not a power of 2.

Proof. By Lemma 4, it is sufficient to show that $\mathscr{P}_{n} \subseteq \mathscr{H}_{n}$. Let $f \in \mathscr{P}_{n}$ and again write $n=q m$. Then $f$ has a strongly simple orbit of period $n$, by Theorem 1 . Let the blocks $B_{1}, \ldots, B_{m}$ of this orbit be numbered so that the midpoint of $B_{k}$ is $f^{k-1}(z)$ for $1 \leqslant k \leqslant m$. For definiteness suppose that the points of $B_{1}$ are

$$
z_{q}<z_{q-2}<\cdots<z_{3}<z_{1}<z_{2}<\cdots<z_{q-1},
$$

where $z_{j}=f^{(j-1) m}(z)$. Choose an interval $J_{q-1}=\left[x_{q-1}, y_{q-1}\right] \subseteq\left[z_{q}, z_{q-2}\right]$ so that $f^{k}\left(J_{q-1}\right) \subseteq\left\langle f^{k}\left(z_{q}\right), f^{k}\left(z_{q-2}\right)\right\rangle$ for $1 \leqslant k \leqslant m$ and $f^{m}\left(y_{q-1}\right)=z_{2}, f^{m}\left(x_{q-1}\right)=z_{1}$. Next define intervals $J_{q-2}, \ldots, J_{1}$ inductively by choosing $J_{q-i}=\left[x_{q-i}, y_{q-i}\right] \subseteq$ $\left\langle z_{q-i+1}, z_{q-i-1}\right\rangle$ so that $f^{k}\left(J_{q-i}\right) \subseteq\left\langle f^{k}\left(z_{q-i+1}\right), f^{k}\left(z_{q-i-1}\right)\right\rangle$ for $1 \leqslant k<m$, $f^{m}\left(J_{q-i}\right)=J_{q-i+1}$ and $f^{m}\left(y_{q-i}\right)=x_{q-i+1}, f^{m}\left(x_{q-i}\right)=y_{q-i+1}(1<i<q)$. Finally choose an interval $J_{0}=\left[x_{0}, y_{0}\right] \subseteq\left[z_{1}, x_{1}\right]$ so that $f^{k}\left(J_{0}\right) \subseteq\left\langle f^{k}\left(z_{1}\right), f^{k}\left(x_{1}\right)\right\rangle$ for $1 \leqslant k<m, f^{m}\left(J_{0}\right)=J_{1}$ and $f^{m}\left(y_{0}\right)=x_{1}, f^{m}\left(x_{0}\right)=y_{1}$. Thus we have $z_{1} \leqslant x_{0}<$ $y_{0}<x_{1}<y_{1} \leqslant z_{2}$ and $f^{n}\left(x_{0}\right)=z_{1}, f^{n}\left(y_{0}\right)=z_{2}$. Moreover $f^{i m}\left(J_{0}\right)=J_{i}$ for $0<i$ $<q$ and $f^{n}\left(J_{0}\right)=\left[z_{1}, z_{2}\right] \supseteq J_{0}$. Thus $J_{0}$ contains a fixed point of $f^{n}$. Furthermore any such fixed point is a periodic point of $f$ with period $n$, the points in its orbit having the same order along the real line as the points in the orbit of $z$. If $a$ is the greatest fixed point of $f^{n}$ in $J_{0}$, then $f^{n}(x)>x$ for $a<x \leqslant y_{0}$. Since $f^{n}\left(x_{1}\right)=z_{3}$ and $f^{n}\left(y_{0}\right)=z_{2}$, there is a least point $b \in\left(y_{0}, x_{1}\right)$ such that $f^{n}(b)=a$ and a least point $c \in\left(a, y_{0}\right)$ such that $f^{n}(c)=b$. It follows that both (5) and (6) hold, and thus $f \in \mathscr{H}_{n}$. The proof shows in addition that there is a homoclinic point of type (I) and period $n$ whose orbit is strongly simple. Q.E.D.

It is stated without proof by Fedorenko and Sarkovskii [11] that if a map has a periodic point whose period $n$ is not a power of 2 , then it has a homoclinic point of period $n$. Theorem 3 , in conjunction with Lemma 4, establishes a more precise result 
and relates the results of Fedorenko and Šarkovskii to those of Blokh [8] in the same volume.

By combining Lemma 7 with Theorem 2 or Theorem 3 we see that a map $f$ has a homoclinic point if and only if $f^{n}$ is turbulent for some $n>0$. This was already proved by Block [2]. The present proof shows that there are always many homoclinic points of a very simple type.

5. Unimodal maps with negative Schwarzian. A map $f$ is said to be unimodal if it has a unique extremum $c$, so that either $f$ is strictly increasing to the left of $c$ and strictly decreasing to the right of $c$, or vice versa. It is evident that any simple orbit of a unimodal map is also strongly simple. On the other hand, it is possible to construct a map with a unique orbit of period 6 so that this orbit is strongly simple but not unimodal.

For any four distinct points $x_{1}, x_{2}, x_{3}, x_{4}$ on the real line, let

$$
R\left(x_{1}, x_{2}, x_{3}, x_{4}\right)=\left(x_{4}-x_{1}\right)\left(x_{3}-x_{2}\right) /\left(x_{4}-x_{3}\right)\left(x_{2}-x_{1}\right)
$$

denote their cross-ratio. If $f:[a, b] \rightarrow \mathbf{R}$ is continuous and strictly monotonic then, following Preston [18], we say that $f$ has property $R$ if

$$
R\left(f\left(x_{1}\right), f\left(x_{2}\right), f\left(x_{3}\right), f\left(x_{4}\right)\right)>R\left(x_{1}, x_{2}, x_{3}, x_{4}\right)
$$

whenever $a<x_{1}<x_{2}<x_{3}<x_{4}<b$.

It is well known, see Preston [18, Proposition 4.1], that if $f$ is three times differentiable and $f^{\prime}(x) \neq 0$ for all $x \in(a, b)$, then $f$ has property $R$ if and only if the Schwarzian derivative

$$
S f(x)=f^{\prime \prime \prime}(x) / f^{\prime}(x)-\frac{3}{2}\left[f^{\prime \prime}(x) / f^{\prime}(x)\right]^{2}
$$

is negative for all $x \in(a, b)$.

We will have occasion to use the following two results concerning functions with property $R$, which are also given by Preston [18, Lemma 4.1 and Proposition 4.6].

Lemma A. If $f:[a, b] \rightarrow[c, d]$ and $g:[c, d] \rightarrow \mathbf{R}$ are continuous, strictly monotonic functions with property $R$, then $g \circ f:[a, b] \rightarrow \mathbf{R}$ is also a continuous, strictly monotonic function with property $R$.

Lemma B. Let $g:[a, b] \rightarrow \mathbf{R}$ be continuous and strictly monotonic with $g(a) \leqslant a$, $g(b) \geqslant b$ and $g(e)=e$ for some $e \in(a, b)$. If $g$ has property $R$ then $e$ is the only fixed point of $g$ in $(a, b)$ and

$$
g(x)<x \text { for } a<x<e, \quad g(x)>x \text { for } e<x<b .
$$

Let $I=[a, b]$ be the given compact interval. We will denote by $\mathscr{C}$ the class of all continuous maps $f: I \rightarrow \mathbf{R}$ which are unimodal and have property $R$ on both the interval $[a, c]$ and the interval $[c, b]$, where $c \in(a, b)$ is the unique extremum of $f$.

This is the same as the class $\mathscr{C}$ studied by Block and Hart [7], except that here we require only $f \in C^{0}$ instead of $f \in C^{3}$ and we drop the inessential condition $f(b)=f(a)=a$. The much-studied quadratic map $f(x)=\lambda x(1-x)$ on the interval $I=[0,1]$ is a typical element of $\mathscr{C}$. 
It was shown by Block and Hart [7] that if a map $f \in \mathscr{C}$ has an orbit of period $n=q m$ with $q>1$, then it has a homoclinic point of period $m$. We show here that it actually has a homoclinic point of type (II) and period $m$, in the terminology of $\$ 4$. This is then used to show that Theorem 2 takes a much simpler form for maps in $\mathscr{C}$, and that the homoclinic stratification which Block and Hart obtained for maps in $\mathscr{C}$ is a consequence of the turbulence stratification for arbitrary continuous maps.

Proposition 8. $\mathscr{P}_{q m} \cap \mathscr{C} \subseteq \mathscr{H}_{m}^{\#}$ if $q>1$ is odd and $m=2^{s} \geqslant 1$.

Proof. We may assume $q \geqslant 5$, since $\mathscr{P}_{3 m} \subset \mathscr{P}_{q m}$ if $q>3$. Let $f \in \mathscr{P}_{q m} \cap \mathscr{C}$. By Theorem $\mathbf{A}, f$ has a simple orbit of period qm. The unique extremum $c$ of $f$ must lie between the least and greatest points of this orbit. Moreover $c$ cannot be situated between two consecutive blocks, since otherwise $f$ would map each block strictly monotonically onto its image and $g=f^{m}$ would map each block strictly monotonically onto itself, which contradicts $q>2$. Hence $c$ lies between the least and greatest points of some block $B$. We can write $B=\left\{y_{1}, \ldots, y_{q}\right\}$, where $g\left(y_{j}\right)=y_{j+1}$ $(1 \leqslant j<q), g\left(y_{q}\right)=y_{1}$ and, without loss of generality,

$$
y_{q}<y_{q-2}<y_{q-4}<\cdots<y_{1}<y_{2}<\cdots<y_{q-3}<y_{q-1} .
$$

Let $J=[\alpha, \beta]$ be a subinterval of $\left[y_{q}, y_{q-1}\right]$ on which $f$ is strictly monotonic. If $f(\alpha)$ and $f(\beta)$ lie between the least and greatest points of the block $f(B)$, then $f$ is also strictly monotonic on $f(J), f^{2}(J), \ldots, f^{m-1}(J)$ and hence $g$ is strictly monotonic on $J$.

Since $g\left(y_{q}\right)<g\left(y_{q-4}\right)<g\left(y_{q-2}\right), g$ is not strictly monotonic on the interval $\left[y_{q}, y_{q-4}\right]$. It follows that the extremum $c$ of $f$ satisfies $y_{q}<c<y_{q-4}$. Moreover, for one point $y_{j}$ of the two points of the orbit adjacent to $c, f\left(y_{j}\right)$ is an endpoint of the whole orbit. Then $g\left(y_{j}\right)$ is an endpoint of $B$, since $f$ is strictly monotonic on all blocks except $B$. Hence $j \neq q, q-4$, which forces $j=q-2$. If $y_{q-2}<c<y_{q-4}$ choose $z \in\left(c, y_{q-4}\right)$ so that $f(z)=f\left(y_{q-2}\right)$, and if $c<y_{q-2}$ take $z=y_{q-2}$. Then $f$ is strictly monotonic on the interval $\left[z, y_{q-1}\right]$, and so also is $g$.

The interval $\left(y_{1}, y_{2}\right)$ contains a fixed point $x_{0}$ of $g$. Let $x_{1}$ be the greatest point of $\left(y_{q}, y_{1}\right)$ such that $g\left(x_{1}\right)=x_{0}$. Evidently we must have $x_{1}<z$. There exists a unique point $x_{2} \in\left(x_{0}, y_{q-1}\right)$ such that $g\left(x_{2}\right)=x_{1}$, a unique point $x_{3} \in\left(z, x_{0}\right)$ such that $g\left(x_{3}\right)=x_{2}$, and a unique point $x_{4} \in\left(x_{0}, x_{2}\right)$ such that $g\left(x_{4}\right)=x_{3}$. Then $g(x)>x_{0}$ for $x_{1}<x<x_{0}$, by the definition of $x_{1}$, and $g$ maps $\left[x_{0}, x_{2}\right]$ strictly monotonically onto $\left[x_{1}, x_{0}\right]$. Hence $g(x)<x_{0}<g^{2}(x)$ for $x_{0}<x<x_{2}$. Moreover $g^{2}(x)<x_{2}$ for $x_{0}<x<x_{4}$, since $g^{2}$ maps $\left[x_{0}, x_{4}\right]$ strictly monotonically onto $\left[x_{0}, x_{2}\right]$.

The argument up to this point uses only the fact that $f$ is unimodal. On the other hand, by Lemma $\mathrm{A}, g^{2}$ has property $R$ on the interval $\left[x_{3}, x_{4}\right]$. Hence, by Lemma $\mathrm{B}$, $x_{0}$ is the unique fixed point of $g^{2}$ in $\left(x_{3}, x_{4}\right)$ and $x<g^{2}(x)$ for $x_{0}<x<x_{4}$. Since $x_{0}$ is a periodic point of $f$ of period $m$, it now follows that $f \in \mathscr{H}_{m}^{*}$. Q.E.D.

For unimodal maps which do not have property $R$, it is not necessarily true that $\mathscr{P}_{q m} \subseteq \mathscr{H}_{m}^{*}$. The proof of Proposition 8 itself shows how to construct a counterexample.

Proposition 9. $\mathscr{H}_{2} \cap \mathscr{C}=\varnothing$. 
Proof. Let $f \in \mathscr{C}$ and, without loss of generality, assume $f$ has a maximum at its unique extremum $c$. Let $x_{1}<x_{2}$ be an orbit of $f$ of period 2 .

Suppose first that $x_{1}<c$, and hence $x_{2}>c$. For any small $\varepsilon>0$ the interval $\left(x_{1}, x_{1}+\varepsilon\right)$ is mapped by $f$ to an interval $\left(x_{2}, x_{2}+\delta\right)$ and by $f^{2}$ to an interval $\left(x_{1}-\eta, x_{1}\right)$, where $\delta>0, \eta>0$. In the same way the interval $\left(x_{1}-\varepsilon, x_{1}\right)$ is mapped by $f^{2}$ to an interval $\left(x_{1}, x_{1}+\eta^{\prime}\right)$, where $\eta^{\prime}>0$. Thus $x_{1}$ cannot be a homoclinic point of type (I). A similar argument applies to $x_{2}$.

Suppose next that $x_{1} \geqslant c$. The open interval $\left(x_{1}, x_{2}\right)$ contains a fixed point $x_{0}$ of $f$. Moreover, by Lemmas A and B,

$$
f^{2}(x)<x \text { if } x \in\left(x_{1}, x_{0}\right), \quad f^{2}(x)>x \text { if } x \in\left(x_{0}, x_{2}\right) .
$$

The cases $x_{1}=c$ and $x_{1}>c$ now require separate treatment. If $x_{1}=c$ then $\left(x_{1}-\varepsilon, x_{1}\right)$ is mapped by $f$ to $\left(x_{2}-\delta, x_{2}\right)$ and by $f^{2}$ to $\left(x_{1}, x_{1}+\eta\right)$, where $\delta>0$, $\eta>0$. Thus $x_{1}$, and similarly $x_{2}$, cannot be a homoclinic point of type (I). Finally suppose $x_{1}>c$. If $f^{2}(y) \leqslant y$ for some $y \in\left(x_{1}-\varepsilon, x_{1}\right)$ then $f^{2}$ is strictly monotonic on $\left[y, x_{2}\right], f^{2}\left(x_{2}\right)=x_{2}$, and $f^{2}$ has two fixed points $x_{1}, x_{0}$ in $\left(y, x_{2}\right)$, which contradicts Lemma B. We conclude that $f^{2}(x)>x$ for all $x \in\left(x_{1}-\varepsilon, x_{1}\right)$. Similarly we must have $f^{2}(x)<x$ for all $x \in\left(x_{2}, x_{2}+\varepsilon\right)$. Thus again $x_{1}$ and $x_{2}$ cannot be homoclinic points of type (I). Q.E.D.

The argument used to prove Proposition 9 can be generalized in the following way.

Proposition 10. If $f \in \mathscr{C}$ has a homoclinic point of type (I) and of period $2^{s}$, where $s>1$, then the orbit of this point is not simple.

Proof. Let $x_{1}<\cdots<x_{m}$ be an orbit of $f$ of period $m=2^{s}$ containing a homoclinic point of type (I). Assume, on the contrary, that this orbit is simple. The extremum $c$ cannot lie in one of the intervals $\left(x_{1}, x_{2}\right),\left(x_{3}, x_{4}\right), \ldots,\left(x_{m-1}, x_{m}\right)$, because then $f$ would be decreasing at an odd number of points $x_{j}$ and hence $f^{m}$ would be decreasing at every $x_{j}$, which is impossible if $x_{j}$ is a homoclinic point of type (I). Consequently, $f$ is strictly monotonic on each of these intervals and they are permuted in some cyclic order by $f$. Hence $f^{m / 2}$ maps each of these intervals homeomorphically onto itself with reversal of orientation. Thus $f^{m / 2}$ has a fixed point $z_{j} \in\left(x_{j}, x_{j+1}\right)$ for every odd $j$. Moreover, by Lemmas A and B, for every odd $j$

$$
f^{m}(x)<x \quad \text { if } x \in\left(x_{j}, z_{j}\right), \quad f^{m}(x)>x \quad \text { if } x \in\left(z_{j}, x_{j+1}\right) .
$$

If $c=x_{i}$ for some $i$ then $f$ is strictly monotonic in a neighborhood of each $x_{j}$ with $j \neq i$, but $f$ maps a whole neighborhood of $x_{i}$ onto a one-sided neighborhood of $f\left(x_{i}\right)$. Hence $f^{m}$ maps a whole neighborhood of every $x_{j}$ onto a one-sided neighborhood of $x_{j}$. It follows that if $j$ is odd a left neighborhood of $x_{j}$ is mapped by $f^{m}$ onto a right neighborhood of $x_{j}$, and if $j$ is even a right neighborhood of $x_{j}$ is mapped by $f^{m}$ onto a left neighborhood of $x_{j}$. In conjunction with the inequalities displayed above this shows that no $x_{j}$ is a homoclinic point of type (I). We reach the 
same conclusion if $c \neq x_{i}$ for every $i$ by a similar argument to that used in the proof of Proposition 9. Q.E.D.

We can now establish the main result of this section.

THEOREM 4. In $\mathscr{C}, \mathscr{H}_{2^{s-1}}^{\#}=H\left(2^{s-1}\right)=\mathscr{T}_{2^{s}}$ for any $s \geqslant 1$.

Proof. In general we have, by Lemma $7, \mathscr{H}_{2^{s-1}}^{\#} \subseteq H\left(2^{s-1}\right) \subseteq \mathscr{T}_{2^{s}}$. Therefore, by Theorem 2, it is sufficient to show that, in $\mathscr{C}, \mathscr{H}_{2^{s}} \subseteq \mathscr{H}_{2^{s-1}}^{\#}$. For $s=1$ this follows at once from Proposition 9. Suppose $s>1$. Then $\mathscr{H}_{2^{s}} \subseteq \mathscr{P}_{3 \cdot 2^{s-2}}$, by Proposition 10 and Theorem $\mathrm{C}$, and $\mathscr{P}_{3 \cdot 2^{s-2}} \subseteq \mathscr{P}_{3 \cdot 2^{s-1}} \subseteq \mathscr{H}_{2^{s-1}}^{\#}$, by Sarkovskii's theorem and Proposition 8. Q.E.D.

The conclusion of Theorem 4 need not hold for maps which are unimodal but do not have property $R$. For example, let $f$ be a smooth map such that $f(0)=4$, $f(1)=5, f(2)=4, f(3)=3, f(4)=2, f(5)=0$, and which is strictly monotonic on the intervals between these points. Then $f$ is unimodal and $f^{2}$ is turbulent, but $f$ has no homoclinic fixed point. Similarly, the conclusion of Theorem 4 need not hold for smooth maps with negative Schwarzian which are not unimodal.

The homoclinic stratification which Block and Hart [7] obtained for maps in the class $\mathscr{C}$ follows at once from the turbulence stratification and Theorem 4 . In the same way Lemma 6 implies their result that $H\left(2^{s-1}\right)$ is a closed set for $C^{3}$ maps in $\mathscr{C}$.

\section{REFERENCES}

1. R. L. Adler, A. G. Konheim and M. H. McAndrew, Topological entropy, Trans. Amer. Math. Soc. 114 (1965), 309-319.

2. L. Block, Homoclinic points of mappings of the interval, Proc. Amer. Math. Soc. 72 (1978), 576-580.

3. 391-398.

4. , Simple periodic orbits of mappings of the interval, Trans. Amer. Math. Soc. 254 (1979), $333-336$.

5. L. Block, J. Guckenheimer, M. Misiurewicz and L. S. Young, Periodic points and topological entropy of one-dimensional maps, Lecture Notes in Math., vol. 819, Springer-Verlag, Berlin and New York, 1980, pp. 18-34.

6. L. Block and D. Hart, The bifurcation of homoclinic orbits of maps of the interval, Ergodic Theory Dynamical Systems 2 (1982), 131-138.

7. Stratification of the space of unimodal interval maps, Ergodic Theory Dynamical Systems 3 (1983), 533-539.

8. A. M. Blokh, An interpretation of a theorem of A. N. Šarkouskii, Oscillation and Stability of Solutions of Functional-Differential Equations, Inst. Mat. Akad. Nauk Ukrain. SSR, Kiev, 1982, pp. 3-8. (Russian)

9. W. A. Coppel, Šarkouskii-minimal orbits, Math. Proc. Cambridge Philos. Soc. 93 (1983), 397-408.

10. M. Denker, C. Grillenberger and K. Sigmund, Ergodic theory on compact spaces, Lecture Notes in Math., vol. 527, Springer-Verlag, Berlin and New York, 1976.

11. V. V. Fedorenko and A. N. Šarkovskii, Stability properties of a dynamical system with a homoclinic trajectory, Oscillation and Stability of Solutions of Functional-Differential Equations, Inst. Mat. Akad. Nauk Ukrain. SSR, Kiev, 1982, pp. 111-113. (Russian)

12. C.-W. Ho, On the structure of the minimum orbits of periodic points for maps of the real line, Preprint, Southern Illinois Univ., Edwardsville.

13. , On Block's condition for simple periodic orbits of functions on an interval, Trans. Amer. Math. Soc. 281 (1984), 827-832.

14. A. Lasota and J. A. Yorke, On the existence of invariant measures for transformations with strictly turbulent trajectories, Bull. Acad. Polon. Sci. Sér. Sci. Math. Astronom. Phys. 25 (1977), 233-238. 
15. T. Y. Li, M. Misiurewicz, G. Pianigiani and J. A. Yorke, Odd chaos, Phys. Lett. A 87 (1982), 271-273.

16. __ No division implies chaos, Trans. Amer. Math. Soc. 273 (1982), 191-199.

17. M. Misiurewicz and W. Szlenk, Entropy of piecewise monotone mappings, Studia Math. 67 (1980), 45-63.

18. C. Preston, Iterates of maps on an interval, Lecture Notes in Math., vol. 999, Springer-Verlag, Berlin and New York, 1983.

19. A. N. Šarkovskii, Coexistence of cycles of a continuous mapping of the line into itself, Ukrain. Mat. $\check{Z}$. 16 (1964), 61-71. (Russian)

20. P. Śtefan, $A$ theorem of Šarkoüskii on the existence of periodic orbits of continuous endomorphisms of the real line, Comm. Math. Phys. 54 (1977), 237-248.

Department of Mathematics, University of Florida, Gainesville, Florida 32611

Department of Mathematics, I.A.S., Australian National University, Canberra, A.C.T. 2601, Australia 